\title{
Evaluation of a Mobile Station Electronic Health Record on Documentation Compliance and Nurses' Attitudes
}

\author{
Mei-Wen Wu${ }^{1}$, Ting-Ting Lee ${ }^{2 *}$, Tzu-Chuan Tsai ${ }^{1}$, Chin-Yi Huang1, Francis Fu-Sheng Wu ${ }^{3}$, \\ Mary Etta Mills \\ ${ }^{1}$ Changhua Christian Hospital, Changhua City, Taiwan \\ ${ }^{2}$ National Taipei University of Nursing and Health Sciences, Taipei, Taiwan \\ ${ }^{3}$ University of Otago, Wellington, New Zealand \\ ${ }^{4}$ University of Maryland, Baltimore, USA \\ Email: *tingting@ntunhs.edu.tw
}

Received 20 June 2015; accepted 20 July 2015; published 24 July 2015

Copyright (C) 2015 by authors and Scientific Research Publishing Inc.

This work is licensed under the Creative Commons Attribution International License (CC BY). http://creativecommons.org/licenses/by/4.0/

(c) (i) Open Access

\section{Abstract}

Electronic Health Record (EHR) Systems have been adopted by healthcare organizations for documentation of patient care. Often these information systems are embedded in mobile nurse stations. As part of assessing the impact of this technology it is important to determine the effect it has on charting compliance and user acceptance. Data were collected at a medical center in Taiwan in two stages. The first stage involved use of a 28-item medical review tool to measure charting compliance in 99 charts before and after implementation of the EHR system. In stage two, a survey was conducted with 709 nurse users to determine their level of mobile EHR acceptance 3 months after this documentation technology was initiated. Results demonstrated that EHR significantly improved documentation compliance in standardized data entry format (name, date, time), abbreviation, content correction/revision, patient care needs, and care goals. Analysis of data from the five categories of a user acceptance survey revealed the following results (based on a 4-point Likert scale): patient care (2.92), nursing efficiency (2.78), education and training (2.98), usability (2.61), and usage benefits (2.87). The study concluded that use of mobile nurse stations with EHR can improve documentation compliance and that although frequent system downtime needs improvement, nurses generally have positive attitudes toward this technology application.

\section{Keywords}

Documentation Compliance, Information System, Mobile Nursing Station, Nurse Attitudes, Technology

\footnotetext{
${ }^{*}$ Corresponding author.
} 


\section{Introduction}

Healthcare organizations have adopted an electronic health record (EHR) system in an effort to improve quality and continuity of patient care [1]. As a documentation tool, the quality of an EHR might, in part, be measured by content completeness (documented items) or content quality (documented based on care theory or nursing process) [2]-[4]. While some quality reviews have noted improved charting compliance on both quantitative and qualitative indicators, it has been suggested that additional factors such as users' acceptance could also impact the use of this technology [5] [6]. As nurses are required to utilize technology as part of their patient care and documentation process, identification of usage outcomes could benefit ongoing system design and development.

To increase documentation convenience and quality, nurses prefer an information system that can provide real-time data entry without a paper format [7] [8]. Mobile nursing stations equipped with an EHR could serve this purpose by providing instant patient history or nursing assessment to assist nurses' decision making or to improve charting care process and outcomes. Nurses acceptance of technology use has been reported as a key factor in the success of technology implementation [9] [10]. However, the correlation between using an EHR and its effect on documentation quality is unclear [11]. There is no standard outcome measurement of an EHR system due to the varied design and development in each care facility, however, user acceptance and documentation compliance could be major determinants for the success of EHR implementation [12] [13]. Therefore, the purpose of this study was to explore documentation compliance and nurse attitudes toward an EHR system which was designed for use on mobile nursing stations.

\section{Literature Review}

\subsection{Documentation Compliance}

Due to the development of information technology (IT), EHRs are replacing traditional paper records. Nursing documentation is an essential aspect of the care process in that it can be one source of identifying the quality of patient care [7] [8]. In general, a complete nursing care record comprises major nursing process steps: assessment, diagnosis, identification of outcomes, planning, implementation, and evaluation [14]. In practice, the patient assessment and diagnostic content are often not completely documented; thus, using an appropriate record structure and guidelines is recommended [15] [16]. The EHR system has been shown to increase the quality of nursing records, improve the integrity of nursing interventions, and reduce documentation variation [3] [8] [17].

Simpson indicated that errors in patient records are a primary cause of one-third of nursing incident events [18]. Nurses often chart their patient care records at the end of their shifts, which is a practice that increases the probability of documentation errors. Therefore, real-time documentation is critical, and the use of mobile technologies better ensures that the content of patient records is accurate [1]. Mobile technology improves compliance with nursing documentation standards because of its real-time data-entry function [8]. Nursing care plans contain nursing diagnoses targeting specific patient problems, and use of a mobile nursing documentation system can enable nurses to revise or retrieve updated care plans that could meet each patient's care needs to improve care quality [7].

With a computerized menu, nurses can complete the documentation process effectively and efficiently. A study based on the closed medical review tool of the Joint Commission on Accreditation of Healthcare Organizations (JCAHO) has shown that after 3 months of system implementation, documentation compliance and quality of patient records increased [8]. The other study [2] examined the effect of computerization on the quality of patient care records by analyzing the case histories of 240 patients who were hospitalized over a three day period. The study revealed that a standardized design of nursing process in the EHR system increased compliance with patient record documentation. Another study [3] compared documentation content and completeness between handwritten, preprinted and computerized nursing care plans. Although computerized nursing care plans increased the documentation of signs and symptoms, related factors and nursing interventions, the mean number of nursing diagnoses per patient did not change.

\subsection{Nurse Attitudes toward Technology Use}

Applying information technology could increase both the standard of care and the patient management process to ensure patient safety, data confidentiality, medication error prevention, information integration, and interdisciplinary communication [19]-[21]. Because of the complexity of patient scenarios, it is impossible to design a 
single system that satisfies the various situations encountered in nursing care. Therefore, other factors such as end user training, hardware devices and software design, workflow change, and user support should be considered when evaluating nurses' technology adoption process [22]. The success of implementing information technology is often based on user acceptance and satisfaction [21]. The factors affecting nurse attitudes toward the EHR system can be categorized into the following five domains: patient care, nursing efficiency, education/ training, usability, and usage benefit.

For the purpose of patient care, standardized care plans with certain nursing diagnoses provided by the information system can be a care guideline for novice nurses, however, experienced nurses prefer to use their own judgment in selecting nursing diagnoses and individualizing care plans [9]. Traditionally, nursing care plans were printed as paper copies, and nurses could choose certain nursing diagnoses first, and then fill out the related factors and illness characteristics to match patient conditions. However, since the EHR has become a required format, nurses cannot diagnose patient problems before identifying illness characteristics and related factors based on physical assessment. This reversed documentation process might cause a change in nurses' acceptance. An EHR that provides accessible nursing diagnoses can assist nurses' decision making for the creation of care plans [9].

Charting and data communication time, time with patients, and structure of work are factors that contribute to nursing efficiency [20]. Studies have shown that nurses spend approximately $19 \%$ to $30 \%$ of their daily working hours in patient record documentation [20] [23]. Yet use of nursing information systems cannot guarantee a reduction of time spent on documentation. Yee et al. [23] analyzed data based on nurses' self entry while working on their shift routines. Results revealed that nurses' spent 19\% of their time on documentation, but when the electronic medical records system and computerized nursing notes were compared to paper documentation, there was no change in nursing documentation time. Furthermore, when a computer-aided nursing (CAN) diagnosis system was implemented, both users and non-users were comparable in selecting an appropriate diagnosis although users could select the required diagnosis faster than their counterparts [24].

Education and training for nurses in the technology adoption process are indispensable. Issues such as typing skills, computer competency, learning anxiety, and stress from using different data entry formats could lead to users' dissatisfaction [10] [25]. Strategic training that targets user attitudes, learning preferences, hardware support, and training design can help nurses with system use [11] [26], for example, a hands-on practice experience and a 24-hour help desk is recommended for user support. It has been suggested that technology alone cannot lead to improvement of patient care or to achieve its desired outcomes. Leadership issues, management support, users skill and attitude assessment all impact the success of technology implementation [3] [5].

Since the use of EHR is intended to alleviate paperwork and nurse charting workload, incorporating usability principles in system design and development can have a significant impact on users' adoption of new technology and work flow change. It is suggested that user-tailored content design, cut and paste functions, data security, and real-time system response increase user acceptance toward technology use [13] [27]. For usage benefits, the availability of nursing interventions to remind nurses to perform standard care and a phrase selection of nursing diagnoses can save time in creating care plans. Furthermore, customizing hot keys such as admission assessments or discharge summaries also increases work efficiency [10]. Patient information is becoming accessible online, thereby raising nurses' awareness to data confidentiality. Work efficiency, time saving, professional image, and data security are all factors affecting nurses' willingness to use technology [25].

Based on the reviewed research, the purpose of this study was to determine the effect of the EHR system on documentation compliance and nurses' attitudes toward mobile EHR use. The research questions for the current study were:

1) Does documentation compliance change following implementation of the EHR system?

2) What are nurses' attitudes toward using the mobile EHR system?

\section{Methods}

\subsection{Design}

This is a pre-post study to explore the effects of an EHR system implemented in a 1200-bed hospital in Taiwan. Data were collected from January to March 2011 and from May to July 2011, respectively. The study was a 3-month pre-post comparison design with a post administered questionnaire survey. The conceptual framework is presented in Figure 1. This study applied the JCAHO closed medical review form 2010 (in Chinese) [8] with 


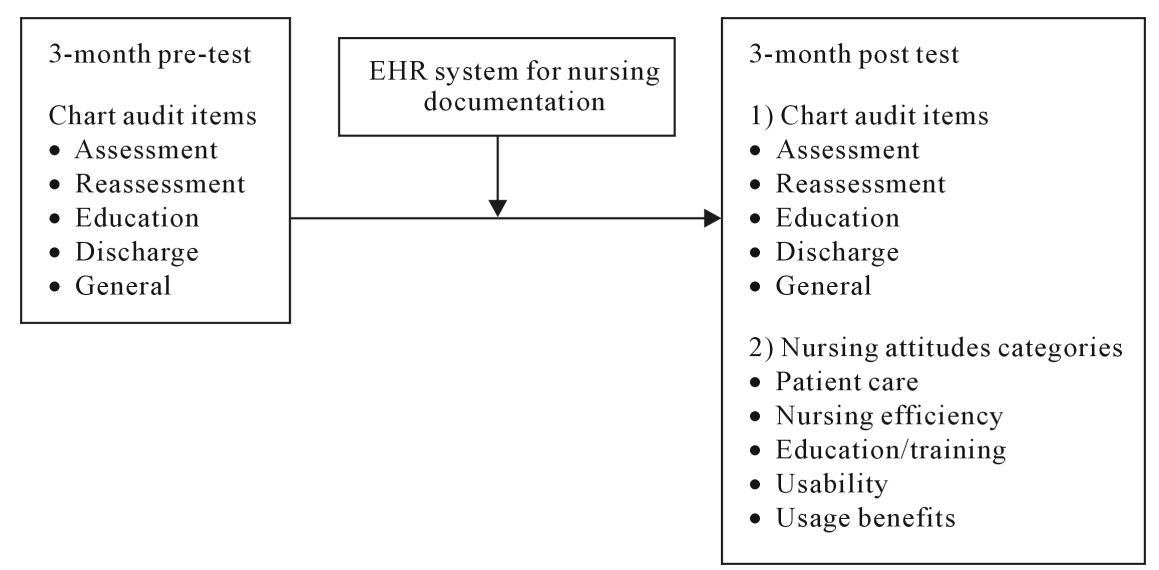

Figure 1. Proposed model of the use the EHR system on documentation.

28 items for measurement of documentation compliance in five main categories (assessment, reassessment, education, discharge, general). User attitudes were measured by a questionnaire with 30 items in five categories (patient care, nursing efficiency, education/training, usability, and usage benefits).

\subsection{Setting}

The hospital information system (HIS) of the study setting included the following sub-systems: a physician order-entry system, a testing and laboratory system, a nursing information system (NIS), and a patient referral system. The mobile nursing documentation information system was integrated with the NIS in 33 inpatient units with 870 nurse users. The EHR for the mobile nursing documentation system includes functions such as admission assessment and care plans with DART (data, action, response, and teaching) format which correspond to the elements of nursing process (assessment, intervention, reassessments, and evaluations).

There are two phases for the use of EHR in nursing documentation on mobile nursing stations: shift reports, medication administration records, and nursing admission notes were available on-line in phase I, and nursing care plans and nursing admission assessments were converted from handwritten to electronic documentation in Phase II; however, the charting of nursing reassessments (other than the first admission assessment) remained in paper form. Nurses could use macro or hot keys to select nursing diagnoses or chart DART on care plans; however, free text design remained available for data entry. For quality control purposes, each nursing diagnosis must be re-evaluated every shift to complete the nursing documentation process.

Although the NIS applications were developed and upgraded, the hardware devices and intranet hotspots retained their original status. To maintain data security, nurses used their staff ID to log into the system and were logged out automatically if there were no additional keyboard or mouse entries within 60 seconds.

\subsection{Data Collection}

The data collection process was divided into two parts: chart review and questionnaire survey. The JCAHO closed medical review form 2010 with three added items (patient care needs, measureable goals, and post-surgical care plan) was used to review care plans with DART format. Each chart with the first three days of three shifts (a total of nine nursing records) of patient admission was reviewed by a research nurse. The 28-item chart audit checklist was divided into the following 10 subcategories: assessment conducted in 24 hours, assessment documented in 24 hours, nutrition assessment, risk assessment, discharge plan, pain assessment, physical assessment, teaching needs assessment, signature/time/date, and others. For each item, "Yes" indicated "compliance" and was scored 1, "No" indicated "non-compliance" and was scored 0, and "N/A" indicated "not applicable" and was not scored.

For attitude survey, the questionnaire was revised from a previous study [9]. Three experts verified the content validity index (CVI) and obtained 0.96 for both content correlation and word clarity. The questionnaire was distributed 3 months after the EHR system was implemented. Each item was rated on a 4-point Likert scale (from 1 "strongly disagree" to 4 "strongly agree"). The total score ranged from 30 to 120 . One open-ended ques- 
tion was provided for nurses to add comments regarding the use of the mobile nursing system.

\subsection{Data Analysis}

To answer the first research question regarding charting compliance, t-test analysis was applied to measure the pre- and post-use of the mobile nursing stations embedded within the EHR system. To answer the second research question regarding nurses' attitude toward the use of the mobile nursing stations embedded with the EHR system, descriptive analysis with means and standard deviations for items in five categories were presented. Comments on this technology adoption process were analyzed based on frequencies of similar and different words or phrases cited by the user.

\section{Results}

For pre- and post-EHR implementation stages, 99 charts were randomly selected in each stage and a total of 198 charts were reviewed and analyzed by t-tests. The results show that the overall documentation compliance significantly increased from $93.04 \%$ to $94.42 \%$ ( $p=0.039$ ). The chi-square and Fisher's exact tests of the 28-item nursing record quality assessment are presented in Table 1 . Six items indicated a significant change after the mobile nursing system was implemented: Items \#22 (every record includes a name, date, and time), \#23 (date of entry), \#24 (correct abbreviation), and \#25 (correction or revision) all showed significant improvement ( $p<$ 0.05). However, Items \#26 (patient care needs based on the assessment and reassessment results) and \#27 (measurable goals) both showed a significant decrease after implementing the mobile EHR system.

Six items (functional assessment, discharge planning, informed consent, care decisions, diagnosis/treatment, and patient right for care process) remained 100\% compliance for both pre- and post-audits. For three added new items, two items (care needs and measurable goals) were decreased, but one item (postsurgical care plan) remained unchanged. Other items were reviewed with varied compliance rates, however, the changes are not statistically significant.

Seven hundred and nine nurses answered the 870 distributed questionnaires for a response rate as $88 \%$. The average total score was 85.06 (out of 120) which was equivalent to 72.1 (out of 100). All the scoring items from the survey are presented in Table 2. The averages of patient care, nursing efficiency, education/training, usability, and usage benefits were 2.92, 2.78, 2.98, 2.61, and 2.87 (on 4-point Likert scale) respectively. Among these five survey categories, education/training revealed the highest scores, and Items 13 (teaching strategies), 17 (problem-solving support), and 18 (training time) all showed an overall score above or equal to 3.0, indicating that users are most satisfied with education and training to increase their problem-solving skills. However, the category of usability showed the lowest average score and item 23, "the system is always working", showed the lowest score of 1.88 in the scale, indicating that users are the most dissatisfied with working EHR on the mobile nursing station.

The user comments are tabulated in Table 3. There were 91 instances within 5 categories as follows: Network function, system content design, user support, hardware device, and workflow change. For network function, most complaints were about "frequent system downtime" followed by "slow system response time". These two comments seemed go together and were on the top of the list. For the second category "system content design", users viewed the care plan content design as needing improvement for customization. Besides, system links with charting time and physician orders, font size, available nursing diagnoses and interventions as part of system content design were also identified as needing improvement. Few complained about data verification processes but insisted on having hot keys to reduce the data entry process. For the user support category, the need for technical help or typing skills was mentioned most often. As for the hardware device, some described the mobile cart as too heavy and the tangle of wires as not safe. Last, some respondents wrote about the workflow change in using paper and EHR at the same time, and one had difficulty in discharging a CPR (Cardiopulmonary Resuscitation) patient using the documentation system.

\section{Discussion}

In this study, while most of the audited items either remained unchanged or changed without statistically significant results, only six items revealed changes after EHR implementation on mobile nursing carts. Additionally, five of the documentation compliance improvements were under the subcategory of documentation requirements 
Table 1. Pre-post comparison for compliance of medical review items N = 198 charts.

\begin{tabular}{|c|c|c|c|}
\hline & Pre (\%) & Post (\%) & $p$ value \\
\hline 1) Initial nursing assessment ( $<24$ hours) & $97.0 \%$ & $98.0 \%$ & 1.0 \\
\hline 2) Assessment findings documented ( $<24$ hours) & $100.0 \%$ & $98.0 \%$ & 0.497 \\
\hline 3) Patients screened for nutritional risk & $99.0 \%$ & $100.0 \%$ & 1.0 \\
\hline 4) Patients screened for nutritional problems & $94.9 \%$ & $99.0 \%$ & 0.212 \\
\hline 5) Patients screened for further functional assessment & $100.0 \%$ & $100.0 \%$ & --- \\
\hline 6) Patients need functional assessment & $98.0 \%$ & $99.0 \%$ & 1.0 \\
\hline 7) Available discharge planning & $100.0 \%$ & $100.0 \%$ & 1.0 \\
\hline 8) Patients are reassessed & $99.0 \%$ & $100.0 \%$ & 1.0 \\
\hline 9) Patients screened for pain & $98.0 \%$ & $98.0 \%$ & 1.0 \\
\hline 10) Pain assessment referred. & $83.8 \%$ & $77.8 \%$ & $0.279^{\mathrm{b}}$ \\
\hline 11) Treatment response reassessed & $84.8 \%$ & $86.9 \%$ & $0.683^{\mathrm{b}}$ \\
\hline 12) Reassessment intervals & $93.9 \%$ & $92.9 \%$ & $0.774^{\mathrm{b}}$ \\
\hline 13) Documented reassessments & $72.7 \%$ & $82.8 \%$ & $0.087^{\mathrm{b}}$ \\
\hline 14) Demographic, emotional, physical and cognitive assessments & $86.9 \%$ & $91.9 \%$ & $0.248^{\mathrm{b}}$ \\
\hline 15) Patient/family educational needs & $98.0 \%$ & $97.0 \%$ & 1.0 \\
\hline 16) Patients and families understand the informed consent & $100.0 \%$ & $100.0 \%$ & --- \\
\hline 17) Patients/families participate in care decisions & $100.0 \%$ & $100.0 \%$ & --- \\
\hline 18) Patients/families understand diagnosis and treatment & $100.0 \%$ & $100.0 \%$ & --- \\
\hline 19) Patients/families' right to participate in care process. & $100.0 \%$ & $100.0 \%$ & --- \\
\hline 20) Assessment findings for patient education & $100.0 \%$ & $98.0 \%$ & 0.497 \\
\hline 21) Patients/families' educational needs assessment & $89.9 \%$ & $91.9 \%$ & $0.621^{\mathrm{b}}$ \\
\hline 22) Every record includes name, date and time. & $76.8 \%$ & $94.9 \%$ & $<0.001^{\mathrm{b}}$ \\
\hline 23) Date of entry & $81.8 \%$ & $98.0 \%$ & $<0.001^{\mathrm{b}}$ \\
\hline 24) Correct abbreviation & $90.9 \%$ & $100.0 \%$ & 0.003 \\
\hline 25) Correction or revision & $85.9 \%$ & $98.0 \%$ & $0.002^{\mathrm{b}}$ \\
\hline 26) Patient care needs based on the assessments and reassessments results & $89.9 \%$ & $65.7 \%$ & $<0.001^{\mathrm{b}}$ \\
\hline 27) Measurable goals & $92.9 \%$ & $83.8 \%$ & $0.046^{\mathrm{b}}$ \\
\hline 28) Available postsurgical plan of care & $98.0 \%$ & $98.0 \%$ & 1.0 \\
\hline
\end{tabular}

Statistically significance marked 'is Pearson chi-square, others are fisher's Exact test. 
Table 2. Scale Item for nursing attitudes $(n=709)$.

\begin{tabular}{|c|c|c|}
\hline & Mean & SD \\
\hline Patient care & 2.92 & 0.51 \\
\hline 1) Needed nursing diagnosis. & 2.89 & 0.51 \\
\hline 2) Needed care goals & 2.88 & 0.52 \\
\hline 3) Available care data (D: data) & 2.94 & 0.51 \\
\hline 4) Available nursing interventions (A: Action) & 2.99 & 0.50 \\
\hline 5) Available patient education content (T: Teaching) & 2.92 & 0.50 \\
\hline 6) Specific care evaluation (R: Response) & 2.92 & 0.49 \\
\hline Nursing efficiency & 2.78 & 0.66 \\
\hline 7) Decrease charting time. & 2.93 & 0.75 \\
\hline 8) Time with patients. & 2.65 & 0.74 \\
\hline 9) Data communication time. & 2.70 & 0.67 \\
\hline 10) Tracking patient problems. & 2.88 & 0.60 \\
\hline 11) Solving patient problems. & 2.75 & 0.59 \\
\hline 12) Care individualization. & 2.77 & 0.58 \\
\hline Education/training & 2.98 & 0.53 \\
\hline 13) Appropriate teaching strategies & 3.03 & 0.51 \\
\hline 14) Short learning time & 2.89 & 0.57 \\
\hline 15) Available technology technician support & 2.88 & 0.60 \\
\hline 16) Available super user support & 2.98 & 0.51 \\
\hline 17) Available problem solving support. & 3.14 & 0.46 \\
\hline 18) Enough training time. & 3.00 & 0.50 \\
\hline Usability & 2.61 & 0.72 \\
\hline 19) Available computer to use & 2.78 & 0.71 \\
\hline 20) Easy read screen display & 2.75 & 0.69 \\
\hline 21) Comfortable contrast color & 2.83 & 0.64 \\
\hline 22) Display patient information. & 2.76 & 0.65 \\
\hline 23) System is always working & 1.88 & 0.88 \\
\hline 24) Easy to print when requested & 2.66 & 0.76 \\
\hline Usage benefit & 2.87 & 0.63 \\
\hline 25) Create nursing professional image & 2.80 & 0.68 \\
\hline 26) Maintain consistency of care plan. & 2.93 & 0.57 \\
\hline 27) Ensure data confidentiality & 2.89 & 0.62 \\
\hline 28) Increase documentation completeness & 2.84 & 0.64 \\
\hline 29) Maintain patient privacy & 2.89 & 0.60 \\
\hline 30) Easy access to patient information. & 2.91 & 0.65 \\
\hline
\end{tabular}


Table 3. Summary of nurses responses to the open-ended question concerned issues $(\mathrm{n}=91)$.

Frequency

\begin{tabular}{|c|c|}
\hline \multicolumn{2}{|l|}{ Network function } \\
\hline Frequent system downtime & 51 \\
\hline Slow system response time & 6 \\
\hline \multicolumn{2}{|l|}{ System content design } \\
\hline Cannot change or revise the standard care plans & 5 \\
\hline Automated presented charting time and physician orders & 4 \\
\hline Font is too small to read & 3 \\
\hline Nursing goals are insufficient & 2 \\
\hline Nursing interventions are insufficient. & 2 \\
\hline Predetermined content is not specialized & 2 \\
\hline Document verified procedure is inconvenient & 1 \\
\hline Set hot keys to reduce typing & 1 \\
\hline \multicolumn{2}{|l|}{ User support } \\
\hline Insufficient technical support & 3 \\
\hline Typing software needed & 2 \\
\hline Typing is slow & 2 \\
\hline \multicolumn{2}{|l|}{ Hardware device } \\
\hline Tangle of wires is unsafe & 2 \\
\hline Mobile cart is too heavy & 2 \\
\hline \multicolumn{2}{|l|}{ Workflow change } \\
\hline Difficult to compare paper and electronic records at the same time & 2 \\
\hline CPR patients could not be discharged until their documentation is done & 1 \\
\hline
\end{tabular}

which included name, date, time, abbreviation, and content revision. These are mandatory items and meet the regulatory requirements. In contrast to manual data entry, each EHR data entry is automatically timed and dated, and each revision can be easily identified. The essential features of the EHR system were the standardized care plans, nursing interventions, and hot keys. The hot keys were chosen based on frequently used words, phrases, and sentences for documentation that aid in the efficiency of data entry [10] [25]. Researchers have suggested that by improving the design of the content, such as a standard format, the overall documentation quality could be increased [15] [16] [18]. A study on cardiac arrest documentation with inconsistent findings suggested that the introduction of a national template can not only capture the relevant data content but also provide evidence of care competence [28].

Standardized document forms allow users to input nursing interventions easily and provide incentives for users to frequently update care plans which could result in a complete nursing document and improve healthcare team communication [2]. However, in this study, the value of Item 26, "patient care needs based on assessment and reassessment results", decreased after the EHR system was implemented. User feedback indicated that the reassessment forms were not electronic and were still in paper form. This task of comparing paper physical assessments and electronic admission assessments was difficult for nurses to review patient status. Therefore, when implementing the EHR system, it is important that the entire nursing workflow be consistent to avoid separating paper and electronic records. 
Item 27, "measurable goals", also showed a significant decrease after implementing the mobile nursing documentation system. Compared with the pre-implementation stage, in which nurses manually wrote care goals as they preferred, after EHR implementation, nurses put feedback such as cannot change or revise care goals. These comments show that if the predetermined contents provided by the mobile nursing information system do not meet the specific needs of patient care, the charting compliance is inadvertently decreased. This contradicts the finding suggesting that a standardized form increases user efficiency, however, it also revealed that technology alone could not achieve the desired outcomes unless the content design or workflow has been changed to meet users' need as well [2]. The results showed that commonly used care goals should be incorporated in the design of the phrasing and content in an EHR system. This further indicates that the users valued the system performance in providing individualized inputs and real-time updated patient status information.

The survey results of nurses' attitudes toward the mobile nursing station showed that "education/training" received the highest group mean score of 2.98, and three items (teaching strategies, problem-solving support, and training time) scored above or equal to 3.0 in this category. One possible reason is that in the study setting, the mobile nursing station had been implemented for shift reporting and medication administration [8]. Nurses were familiar with these tasks and could incorporate them into their daily practice. Although most users responded positively toward the education/training category, very few commented that they still needed technical support and complained about typing skills. While using familiar information systems could smooth the technology adoption process for nurses, educational preparation such as school programs designed for application courses could prepare nursing students with technology-related skills to increase their care proficiency prior to entering the clinical setting [27].

The group mean of the patient care category scored higher than 2.9. This implies that the care plan structure remained the same when DART was converted from paper to electronic format. Nurses did not need to adjust their documentation habits or workflow, which eased the adoption process [9]. In the nursing efficiency category, charting time was rated the highest, indicating that nurses could instantly access the EHR integrated patient data, such as vital signs, medications and test results, which increased their work efficiency. Although "time with patients" was rated positively (2.65), this item scored the lowest in this category, possibly because the purpose of EHR is to integrate patient information for clinical decision-making processes and is unlike patient care technology devices which streamline their workflow and increase time with patients.

The "usage benefit" category also scored a group average mean near 2.9, indicating nurses highly value consistent care plans and accessible patient information, patient privacy and data confidentiality. Although electronic documentation use involves applying shared information to improve care quality, nurses have become aware of patient privacy and data security [19]. As nursing stations become more mobile, private information, such as personal identification numbers, socio-economic status, and treatment outcomes or responses, could be jeopardized in the public areas of a hospital. A login requiring a user ID card and a screen that automatically locks after 60 seconds of lag time are strategies that ensure patient data confidentiality.

The usability category received the lowest average score, and the item "the system is always working" had the lowest score in the survey. Most users commented on the unstable and disconnected information system. This instability occurred because the mobile nursing documentation system was situated on a cart that used a wireless intranet. The hospital's two-year-old wireless network was unable to accommodate the new mobile nursing information system. This problem was exacerbated when the accumulated data slowed the network response time. One study [4] investigated the effect of real-time feedback of charting reminders to improve the documentation quality for the emergency department, and nurses preferred the flexibility of documenting the content at a time which is best for them. Therefore, a network reassessment must be performed prior to implementing a new system to ensure system performance and user satisfaction.

\section{Study Limitations}

This study was conducted in a single hospital with their home developed EHR system embedded in the mobile nursing stations without a control group. Therefore, the results interpretation needs to be considered when applied to other healthcare settings. In addition, personal profile variables such as age, education, nursing experiences, computer skills or informatics literacy that could affect attitudes toward technology adoption or charting behaviors were not explored, although clinical information systems should be designed for all clinicians, regardless of their demographic background. Therefore, future studies could examine the nurse' cognitive loading 
or charting behavior related to the technology adoption process, and propose education strategies to smooth this transition stage.

\section{Conclusion}

In conclusion, implementing a mobile EHR system can increase nurses' charting compliance. Although nurses may possess a positive attitude toward this technology adoption process, the manual assessment paper system reduced the efficiency of patient reassessments. In addition, because mobile technology was used, the network speed was slow and requires improvement. The study results showed that a hospital that is gradually implementing EHR systems should perform network reassessments and improve care plan content design before implementing a new technology to ensure that users can perform daily practice smoothly and the system can maintain function despite the increased network flow.

\section{References}

[1] Häyrinen, K., Saranto, K. and Nykänen, P. (2008) Definition, Structure, Content, Use and Impacts of Electronic Health Records: A Review of the Research Literature. International Journal of Medical Informatics, 77, 291-304. http://dx.doi.org/10.1016/j.ijmedinf.2007.09.001

[2] Mahler, C., Ammenwerth, E., Wagner, A., Tautz, A., Happek, T., Hoppe, B. and Eichstädter, R. (2007) Effects of a Computer-Based Nursing Documentation System on the Quality of Nursing Documentation. Journal of Medical System, 31, 274-282. http://dx.doi.org/10.1007/s10916-007-9065-0

[3] Thordoddsen, A., Ehnfors, M. and Ehrenberg, A. (2011) Content and Completeness of Care Plans after Implementation of Standardized Nursing Terminologies and Computerized Records. Computers, Informatics, Nursing, 29, 599-607. http://dx.doi.org/10.1097/NCN.0b013e3182148c31

[4] Nielsen, G., Peschel, L. and Burgess, A. (2014) Essential Documentation Elements: Quality Tool for the Emergency Department Nurse. Advanced Emergency Nursing Journal, 36, 199-205. http://dx.doi.org/10.1097/TME.0000000000000020

[5] Alexander, S., Frith, K.H., O’Keefe, L. and Hennigan, M.A. (2011) Implementation of Customized Health Information Technology in Diabetes Self Management Programs. Clinical Nurse Specialist, 25, 63-70. http://dx.doi.org/10.1097/NUR.0b013e31820aefd6

[6] Smith, D., Morris, A. and Janke, J. (2011) Nursing Satisfaction and Attitudes with Computerized Software Implementation: A Quasi-Experimental Dtudy. Computes, Informatics, Nursing, 29, 245-250. http://dx.doi.org/10.1097/NCN.0b013e3181f9dcfa

[7] Hsu, S.C., Liu, C.F., Weng, R.H. and Chen, C. J. (2013) Factors Influencing Nurses’ Intentions toward the Use of Mobile Electronic Medical Records. Computers, Informatics, Nursing, 31, 124-132. http://dx.doi.org/10.1097/NXN.0b013e318270100b

[8] Wu, M.W., Lee, T.T., Tsai, T.C., Lin, K.C., Huang, C.Y. and Mills, M.E. (2013) Evaluation of a Mobile Shift Report System on Nursing Documentation Quality. Computers, Informatics, Nursing, 31, 85-93. http://dx.doi.org/10.1097/NXN.0b013e318266cac3

[9] Lee, T.T., Mills, M.E. and Lu, M.H. (2009) The Multimethod Evaluation of a Nursing Information System in Taiwan. Computers, Informatics, Nursing, 27, 245-253. http://dx.doi.org/10.1097/NCN.0b013e3181a91b90

[10] Lee, T.T. (2008) Nursing Information: Users' Experiences of a System in Taiwan One Year after Its Implementation. Journal of Clinical Nursing, 17, 763-771. http://dx.doi.org/10.1111/j.1365-2702.2007.02041.x

[11] Urquhart, C., Currell, R., Grant, M.J. and Hardiker, N.R. (2009) Nursing Record Systems: Effects on Nursing Practice and Healthcare Outcomes. Cochrane Database of Systematic Reviews, No. 1, Article No. CD002099. http://dx.doi.org/10.1002/14651858.cd002099.pub2

[12] McDowell, D.E., Dillon, T.W. and Lending, D. (2008) Perceived Quality Benefits Influenced by Usefulness and Documentation Accuracy of Information Systems. Computers, Informatics, Nursing, 26, 350-357. http://dx.doi.org/10.1097/01.NCN.0000336463.72069.7c

[13] Bennett, K.J. and Steen, C. (2010) Electronic Medical Record Customization and the Impact Upon Chart Completion Rates. Family Medicine, 42, 338-342.

[14] American Nurses Association (2008) Nursing Informatics: Scope and Standards of Practice. NursesBooks, Silver Spring.

[15] Blumenthal, D. and Tavenner, M. (2010) The "Meaningful Use” Regulation for Electronic Health Records. New England Journal of Medicine, 363, 501-504. http://dx.doi.org/10.1056/NEJMp1006114 
[16] Centers for Medicare \& Medicaid Services (2010) EHR Incentive Programs. http://www.cms.gov/Regulations-and-Guidance/Legislation/EHRIncentivePrograms/index.html

[17] Gunningberg, L., Dahm, M.F. and Ehrenberg, A. (2008) Accuracy in the Recording of Pressure Ulcers and Prevention After Implementing an Electronic Health Record in Hospital Care. Quality \& Safety in Health Care, 17, $281-285$. http://dx.doi.org/10.1056/NEJMp1006114

[18] Simpson, R.L. (2005) Patient and Nurse Safety: How Information Technology Makes A Difference. Nursing Administration Quarterly, 29, 97-101. http://dx.doi.org/10.1097/00006216-200501000-00015

[19] Kuo, Y.H., Lee, T.T., Mills, M.E. and Lin, K.C. (2012) The Evaluation of A Web-Based Incident Reporting System. Computers, Informatics, Nursing, 30, 386-394. http://dx.doi.org/10.1097/NXN.0b013e31825106ea

[20] Huang, H.Y. and Lee, T.T. (2011) Impact of Bar-Code Medication Administration on Nursing Activity Patterns and Usage Experience in Taiwan. Computers, Informatics, Nursing, 29, 554-563. http://dx.doi.org/10.1097/NCN.0b013e31820662c1

[21] Yui, B.H., Jim, W.T., Chen, M., Hsu, J.M., Liu, C.Y. and Lee, T.T. (2012) Evaluation of Computerized Physician Order Entry System-A Satisfaction Survey in Taiwan. Journal of Medical System, 36, 3817-3824. http://dx.doi.org/10.1007/s10916-012-9854-y

[22] Gruber, D., Cummings, G.G., LeBlanc, L. and Smith, D.L. (2009) Factors Influencing Outcomes of Clinical Information Systems Implementation: A Systematic Review. Computers, Informatics, Nursing, 27, 151-163. http://dx.doi.org/10.1097/NCN.0b013e31819f7c07

[23] Yee, T., Needleman, J., Pearson, M., Parkerton, P., Parkerton, M. and Wolstein, J. (2012) The Influence of Integrated Electronic Medical Records and Computerized Nursing Notes on Nurses' Time Spent in Documentation. Computers, Informatics, Nursing, 30, 287-292. http://dx.doi.org/10.1097/nxn.0b013e31824af835

[24] Kurashima, S., Kobayashi, K., Toyabe, S. and Akazawa, K. (2008) Accuracy and Efficiency of Computer-Aided Nursing Diagnosis. International Journal of Nursing Terminologies \& Classifications, 19, 95-101. http://dx.doi.org/10.1111/j.1744-618X.2008.00088.x

[25] Lee, T.T. (2007) Nurses’ Experiences Using a Nursing Information System: Early Stage of Technology Implementation. Computers, Informatics, Nursing, 25, 294-300. http://dx.doi.org/10.1097/01.NCN.0000289166.61863.0b

[26] Husting, P.M. and Cintron, L. (2003) Healthcare Information Systems: Education Lessons Learned. Journal for Nurses in Staff Development, 19, 253-257. http://dx.doi.org/10.1097/00124645-200309000-00008

[27] Brooks, C.L. and Erickson, L.K. (2012) What Is the Solution for Clinical Nurse Educators and the Electronic Medical Record? Teaching and Learning in Nursing, 7, 129-132. http://dx.doi.org/10.1016/j.teln.2012.06.003

[28] Allan, N., Bell, D. and Pittard, A. (2011) Resuscitation of the Written Word: Meeting the Standard for Cardiac Arrest Documentation. Clinical Medicine, 11, 348-352. http://dx.doi.org/10.7861/clinmedicine.11-4-348 